\title{
THE
}

UNIVERSITY

University of Rhode Island

OF RHODE ISLAND

DigitalCommons@URI

10-14-1985

\section{Nightingale and Indekeu Respond}

\author{
M. P. Nightingale \\ University of Rhode Island, nightingale@uri.edu \\ J. O. Indekeu
}

Follow this and additional works at: https://digitalcommons.uri.edu/phys_facpubs

Terms of Use

All rights reserved under copyright.

\section{Citation/Publisher Attribution}

Nightingale, M. P., \& Indekeu, J. O. (1985). Nightingale and Indekeu Respond. Physical Review Letters, 55(16), 1700. doi: 10.1103/PhysRevLett.55.1700

Available at: http://dx.doi.org/10.1103/PhysRevLett.55.1700

This Response or Comment is brought to you for free and open access by the Physics at DigitalCommons@URI. It has been accepted for inclusion in Physics Faculty Publications by an authorized administrator of DigitalCommons@URI. For more information, please contact digitalcommons-group@uri.edu. 
Nightingale and Indekeu Respond: In our Letter ${ }^{1}$ we discussed that the thickness $\hat{l}$ of a wetting layer consisting of a critical phase is determined by a finite-size interaction proportional to $\hat{l}^{1-d}$. We also speculated that the amplitude of this interaction is universal in the sense that for a given universality class it can assume a discrete set of values only. One aspect, viz., universality with respect to the surface fields, was verified analytically for the two-dimensional Ising model and within mean-field theory, and in a renormalizationgroup calculation. The other aspect, viz., universality with respect to the details of the microscopic interactions, has been corroborated by Monte Carlo calculations for simple cubic and bcc Ising models in three dimensions. $^{2}$

In our Letter we also assumed that the noncritical spectator phase, which is in equilibrium with the critical wetting layer, can be replaced by a surface field acting on that layer. Here we should like to point out that the model considered by Lipowsky and Seifert ${ }^{3}$ is sufficiently simple that this basic assumption in our analysis of the wetting layer can be verified explicitly. In the notation of Lipowsky, and Lipowsky and Seifert, we take

$$
\begin{aligned}
f(\phi) & =\frac{1}{2} u^{2} \phi^{4}+h \phi, \quad \phi<M, \\
& =\frac{1}{2} \xi^{-2}(1-\phi)^{2}, \quad \phi>M .
\end{aligned}
$$

Here $h$ is any field that takes the system away from critical end-point coexistence, e.g., $h>0$ for a gravity-thinned layer. One way to calculate the thickness $l$ of the wetting layer is by minimizing of the free energy $F_{\hat{l}}$, which is the sum of the gravitational and finite-size energies: $F_{\hat{l}}=h \hat{l}+W / \hat{l}^{3}$, where ${ }^{1} W=\Delta_{+-}$ $=8 K^{4} / 3 u^{2}$, with $k=\int_{0}^{\infty} d \nu\left(1+\nu^{4}\right)^{-1 / 2}$ if the surface field $h_{1}$ is negative (complete wetting), and $W=\Delta_{0+}=K^{4} / 6 u^{2}$ if $h_{1}=0$ (critical wetting), reflecting that the surface field that acts on the critical layer and represents the spectator phase is positive (and equal to $\left.\xi^{-2}\right)$. One then finds

$$
\hat{l}=(3 W / h)^{1 / 4} \text {. }
$$

Alternatively, one can obtain $\hat{l}$ directly from the order parameter profile $M(z)$ that minimizes the free energy. With the free energy density given by Eq. (1), $M(z)$ differs from Eq. (5) of Lipowsky and Seifert only for $0<z<\hat{l}$, where

$$
\zeta-\delta_{1}=\int_{0}^{m} d \nu\left(1+\nu^{4}\right)^{-1 / 2}
$$

with $m=\left(u^{2} / 2 h\right)^{1 / 4} M$ and $\zeta=\left(2 u^{2} h\right)^{1 / 4} z . \quad \delta_{1}$ is fixed by the boundary condition at $z=0$, and for small $h$ it is found to differ negligibly from $\delta_{1}=K$ if $h_{1}<0$, and from $\delta_{1}=0$ if $h_{1}=0$. Likewise, it turns out that for small $h$ the profile and its derivative match at $\zeta \leq K$. In conclusion, the thickness of the wetting layer in the small- $h$ limit is $2 K$ for $h_{1}<0$ and $K$ for $h_{1}=0$ in the new units of length. In the original units this precisely agrees with Eq. (2), which substantiates the assumption that the spectator phase can be replaced by a surface field.

\section{P. Nightingale}

Physics Department

University of Rhode Island

Kingston, R.I. 02881

J. O. Indekeu

Instituut voor Theoretische Physica

Katholieked Universiteit Leuven

3030 Leuven, Belgium

Received 29 August 1985

PACS numbers: 68.10.-m, 05.70.Jk, 64.70.-p, 75.40.Dy

${ }^{1}$ M. P. Nightingale and J. O. Indekeu, Phys. Rev. Lett. 54, 1824 (1985).

${ }^{2}$ K. K. Mon and M. P. Nightingale, Phys. Rev. B 31, 6137 (1985).

${ }^{3}$ R. Lipowsky and U. Seifert, Phys. Rev. B 31, 4701 (1985). 